\title{
ON THE MODULUS OF CONTINUITY OF ANALYTIC FUNCTIONS
}

\section{A. HINKKANEN ${ }^{1)}$}

\section{Introduction and results}

We shall assume throughout the paper that $G$ is an open set in the plane such that $\partial G$, the boundary of $G$, contains at least two (finite) points and that $f$ is a function continuous in $\bar{G}$, the closure of $G$, and analytic in $G$.

We shall call a non-decreasing continuous function $\mu:[0, \infty) \rightarrow[0, \infty)$ a majorant The function $\mu$ must always satisfy some extra conditions, but these vary.

We consider the following problem. Suppose that

$$
\left|f\left(z_{1}\right)-f\left(z_{2}\right)\right| \leqq \mu\left(\left|z_{1}-z_{2}\right|\right)
$$

for all $z_{1}, z_{2} \in \partial G$. When does (1.1) remain valid for all $z_{1}, z_{2} \in \bar{G}$ ? We prove the following result.

Theorem 1. Let $G$ and $f$ be as above, and let $\mu$ be a majorant such that $\log \mu\left(e^{t}\right)$ is a concave function of $t$ for real $t$ and that

$$
B=\lim _{t \rightarrow 0+} \frac{\log \mu(t)}{\log t} \leqq 1
$$

We set

$$
A=\lim _{t \rightarrow \infty} \frac{\log \mu(t)}{\log t} \leqq B
$$

and assume that

$$
f(z)=o(|z|)
$$

if $A<1$, and that

$$
f(z)=o\left(|z|^{2}\right)
$$

if $A=1$, as $z \rightarrow \infty$ in any unbounded component of $G$. If (1.1) holds for all $z_{1}, z_{2} \in \partial G$, then (1.1) remains valid for all $z_{1}, z_{2} \in \bar{G}$. If (1.1) holds for a fixed $z_{1} \in \partial G$ and all $z_{2} \in \partial G$, then (1.1) remains valid for this $z_{1}$ and all $z_{2} \in \bar{G}$.

1) Research supported by the Osk. Huttunen Foundation, Helsinki. 
If instead of (1.4) or (1.5) we only have

$$
f(z)=o\left(|z|^{q}\right)
$$

for some $q>0$, and if the conclusion of the theorem fails, then $G$ contains a neighbourhood of infinity, and $f$ has a pole at infinity.

We may allow the case $\mu \equiv 0$. For if Theorem 1 has been proved in all other cases, we may apply it with $\mu \equiv \varepsilon$ for an arbitrary positive $\varepsilon$ to deduce that it remains valid if $\mu \equiv 0$. From now on we assume that $\mu$ does not vanish identically. Hence $A \geqq 0$.

The condition (1.2) is natural, for $\mu(t)=t^{B+o(1)}$ as $t \rightarrow 0$, and if $B>1$, then (1.1) cannot be true for all $z_{1}, z_{2} \in G$ unless $f$ is constant.

Tamrazov [2, Theorem 9.3, p. 167] showed that if $\mu$ is a majorant satisfying a growth condition, e.g.

$$
\mu(2 t) \leqq 2 \mu(t), \quad t>0,
$$

and if the conclusion of Theorem 1 for a fixed $z_{1} \in \partial G$ is correct for all bounded Jordan domains $G$, then $\log \mu\left(e^{t}\right)$ is concave. Hence this condition is necessary. The reason behind it is that we want the functions $-\log \mu\left(\left|z-z_{0}\right|\right)$ to be subharmonic for $z \in G$, for any $z_{0} \in \partial G$.

Gehring, Hayman and the author [1, Theorem 1] proved Theorem 1 for $\mu(t)=$ $M t^{\alpha}, M>0,0 \leqq \alpha \leqq 1$. In this case $\log \mu\left(e^{t}\right)=\alpha t+\log M$ is concave, and $A=B=\alpha$. For $0 \leqq A<1$, our Theorem 1 applies to other functions that $\mu(t)=M t^{\alpha}$. However, as we shall show in Section 3 , for $A=1$ we get only the functions $\mu(t)=M t$.

Our growth conditions (1.4) and (1.5) and their dependence on $A$ are the same as $[1,(1.2),(1.3)]$ in the case dealt with in [1]. As remarked in [1, p. 243], the functions $z$ and $z^{2}$, respectively, with $G=\{|z|>1\}$, show that $o$ cannot be replaced by $O$ in (1.4) and (1.5).

Results like Theorem 1 were obtained by Tamrazov [2] for special open sets and for majorants $\mu$ satisfying a growth condition which we take to be (1.7). This is satisfied, for example, by any subadditive $\mu$. Let cap $E$ denote the logarithmic capacity of the compact set $E$. Then Tamrazov's results [2, Theorems 4.1., 6.1, 9.1] can be summarized in a slightly simplified form as follows. Suppose that $G$ is bounded or that $G$ contains a neighborhood of infinity, in which case $f$ is required to remain analytic at $\infty$. Hence $\partial G$ is bounded, and $f$ is also bounded. If (1.1) holds for $z_{1}, z_{2} \in \partial G$, and if $z_{1} \in \partial G, z_{2} \in G$, then

$$
\left|f\left(z_{1}\right)-f\left(z_{2}\right)\right| \leqq 27 \mu\left(\left|z_{1}-z_{2}\right|\right)\left|z_{1}-z_{2}\right|\left(2 \operatorname{cap} E\left(z_{1}, z_{2}\right)\right)^{-1},
$$

where

$$
E\left(z_{1}, z_{2}\right)=\left\{\left|z-z_{1}\right| \leqq \frac{1}{2}\left|z_{1}-z_{2}\right|\right\} \backslash G .
$$

If, in addition, $G$ is simply connected, then

$$
\left|f\left(z_{1}\right)-f\left(z_{2}\right)\right| \leqq C \mu\left(\left|z_{1}-z_{2}\right|\right), \quad z_{1}, z_{2} \in \bar{G},
$$


where $C=108$. If $G$ is suitable, one can use (1.8) together with [2, Lemma 4.1] to deduce that (1.9) holds for some $C$ depending on $G$ but not on $z_{1}$ and $z_{2}$. If $\log \mu\left(e^{t}\right)$ is concave, we can take $C=1$ in (1.9) if (1.7) holds and $\partial G$ satisfies certain capacity density conditions. Moreover, if (1.1) holds for a fixed $z_{1} \in \partial G$ and all $z_{2} \in \partial G$, if $f$ is bounded and if $\log \mu\left(e^{t}\right)$ is concave, then (1.9) holds for this $z_{1}$ and all $z_{2} \in \bar{G}$ with $C=1$, provided that $\partial G$ is thick enough. In this case $\partial G$ need not be bounded.

Theorem 1 generalizes Tamrazov's results when $\log \mu\left(e^{t}\right)$ is concave, since we need no assumption on the capacity density of $\partial G$.

References to other earlier works related to this subject can be found in [1, p. 244] and in [2, p. 141-143].

\section{Lemmas}

Let $u$ be a subharmonic function in the plane. We set

$$
M(r, u)=\sup \{u(z)|| z \mid=r\} .
$$

For all functions $u$ that we shall consider, we have

$$
M(r, u)=O(\log r)
$$

as $r \rightarrow \infty$.

To prove Theorem 1, we need two lemmas. The first lemma follows from [1, Theorem 2].

Lemma 1. Suppose that $u$ is subharmonic, non-negative and not constant in the plane, that (2.1) holds as $r \rightarrow \infty$, and that $u(z)=0$ for some $z$. Then the limit

$$
\beta=\lim _{r \rightarrow \infty} M(r, u) / \log r
$$

exists and $0<\beta<\infty$. Suppose further that there is a component $D$ of the set $\{z \mid u(z)>0\}$ such that $u$ is harmonic in $D$ and possesses there a local conjugate $v$, and that for some $\alpha, 0<\alpha \leqq 1$, and some positive $R$, the function

$$
F(z)=z^{1-\alpha} \exp (u+i v)
$$

remains single-valued in $D \cap\{|z|>R\}$. Then $D$ contains the set $\left\{|z|>R_{0}\right\}$ for some $R_{0}$, and $F$ has a pole of order $\beta+1-\alpha$ at infinity.

Note that any such component $D$ is unbounded.

Our second lemma is a generalization of Tamrazov's result [2, Lemma 4.1, p. 156].

Lemma 2. Let $G$ be an open set with at least one (finite) boundary point, and suppose that

$$
f(z)=o\left(|z|^{2}\right)
$$


as $z \rightarrow \infty$ in any unbounded component of $G$. Then for every positive $t$ we have

$$
\begin{gathered}
\sup \left\{\left|f\left(z_{1}\right)-f\left(z_{2}\right)\right||| z_{1}-z_{2} \mid \leqq t, z_{1}, z_{2} \in \bar{G}\right\} \\
=\sup \left\{\left|f\left(z_{1}\right)-f\left(z_{2}\right)\right||| z_{1}-z_{2} \mid \leqq t, \quad z_{1} \in \partial G, z_{2} \in \bar{G}\right\} .
\end{gathered}
$$

Tamrazov proved Lemma 2 with the additional hypothesis that $\partial G$ is bounded and $f$ is bounded (cf. Section 1).

To prove Lemma 2, we consider a fixed positive $t$, denote the left and right hand sides of (2.4) by $L$ and $R$, respectively, and note that $R \leqq L$. To prove that $L \leqq R$, we may assume that $L>0, R<\infty$, so that $f$ is not constant.

Pick $z_{1}, z_{2} \in G$ such that with $h=z_{2}-z_{1}$ we have $|h| \leqq t$. It suffices to show that with

$$
g(z)=f(z)-f(z+h)
$$

we have $\left|g\left(z_{1}\right)\right| \leqq R$. The function $g$ is defined and continuous in the closure of the non-empty open set

$$
G_{1}=\{z \mid z \in G, z+h \in G\} \subset G
$$

and analytic in $G_{1}$. Further, $g$ is bounded in any compact subset of $\bar{G}_{1}$, and $z_{1} \in G_{1}$. If $z \in \partial G_{1}$, then $z \in \partial G$ or $z+h \in \partial G$, so that $|g(z)| \leqq R$. If $g$ is bounded in $G_{1}$, it follows from the maximum principle that $\left|g\left(z_{1}\right)\right| \leqq R$.

Suppose that $g$ is unbounded, and let $D$ be a component of the open set $\left\{z \in G_{1}|| g(z) \mid>R\right\}$, so that $D$ is unbounded. We set

$$
\begin{gathered}
u(z)=\log |g(z)|-\log R, \quad z \in D, \\
u(z)=0, \quad z \notin D,
\end{gathered}
$$

and note that $u$ is subharmonic and non-constant in the plane, and harmonic and positive in $D$, and possesses a local conjugate in $D$, namely, $\arg g(z)$. By (2.3), the condition (2.1) holds, and we may apply Lemma 1 to $u$ with $\alpha=1$. It follows that $D$ and thus $G$ contains a neighborhood of $\infty$, so that by (2.3), $f$ remains analytic or has a pole of order one at $\infty$. But in both cases $g$ remains bounded at $\infty$, which is against our assumption. Hence $L \leqq R$, and Lemma 2 is proved.

\section{Proof of Theorem 1}

Suppose that the assumptions of Theorem 1 are satisfied and take a fixed $z_{1} \in \partial G$. The function

$$
u_{1}(z)=\log \left|f(z)-f\left(z_{1}\right)\right|-\log \mu\left(\left|z-z_{1}\right|\right)
$$

is subharmonic in $G$ and satisfies by (1.2),

$$
\limsup _{z \rightarrow \zeta} u_{1}(z) \leqq 0
$$


for all $\zeta \in \partial G, \zeta \neq z_{1}$. Further, $u_{1}$ is bounded above in any compact subset of $\bar{G}$ not containing $z_{1}$.

First we show that $u_{1}$ is bounded above in a neighbourhood of $z_{1}$ in $\bar{G}$. Suppose that this is not true, and let $D_{1}$ be a component of the open set $\left\{z|| z-z_{1} \mid<1, z \in G\right.$, $\left.u_{1}(z)>M\right\}$, where $M=\max \left(0, \sup \left\{u_{1}(z)|z \in \bar{G}| z-,z_{1} \mid=1\right\}\right)$. Hence $z_{1} \in \partial D_{1}$.

We set $\varphi(z)=\left(z-z_{1}\right)^{-1}, \varphi(G)=G_{1}, \varphi\left(D_{1}\right)=D_{2}$, and note that $\varphi\left(z_{1}\right)=\infty$, and $0 \notin G_{1}$. We set

$$
\begin{array}{ll}
u(z)=u_{1}\left(\varphi^{-1}(z)\right)-M, & z \in D_{2}, \\
u(z)=0, & z \notin D_{2},
\end{array}
$$

so that $u$ is non-negative, non-constant and subharmonic in the plane and positive in $D_{2}$. Also,

$$
u(z)=\log \left|f\left(z_{1}+1 / z\right)-f\left(z_{1}\right)\right|-\log \mu(1 /|z|)-M, \quad z \in D_{2} .
$$

By (1.2),

$$
-\log \mu(1 /|z|) \leqq(B+o(1)) \log |z| \leqq(1+o(1)) \log |z|
$$

as $z \rightarrow \infty$. Hence (2.1) holds. Now we apply Lemma 1 to $u$ to obtain $0<\beta \leqq B \leqq 1$. In particular, $B>0$.

Choose $\varepsilon$ such that $0<\varepsilon<\beta \leqq B$. Consider the function

$$
u_{2}(z)=\log \left|f\left(z_{1}+1 / z\right)-f\left(z_{1}\right)\right|+(B-\varepsilon) \log |z|-M, \quad z \in D_{2} .
$$

Since $M(r, u) \sim \beta \log r$, since $-\log \mu(1 /|z|) \sim B \log r, r=|z|$, and since

$$
u(z)-u_{2}(z)=-\log \mu(1 /|z|)-(B-\varepsilon) \log |z|, \quad z \in D_{2},
$$

we have $M\left(r, u_{2}\right) \sim(\beta-\varepsilon) \log r \rightarrow \infty$ as $r \rightarrow \infty$. Further,

$$
(B-\varepsilon) \log |z|<-\log \mu(1 /|z|)
$$

when $|z|$ is large enough, so that $u_{2}(z)<0$ if $z \in \partial D_{2}$ and $|z|>R$. We have $u_{2}(z) \leqq M_{2}$ for $|z|=R$, for some positive $M_{2}$, so that

$$
\limsup _{z \rightarrow \zeta} u_{2}(z)-M_{2} \leqq 0
$$

for every finite boundary point $\zeta$ of $D_{3}=D_{2} \cap\{|z|>R\}$. Since $u_{2}$ is unbounded in $D_{3}$, we can find an unbounded component $D_{4}$ of the set $\left\{z \in D_{3} \mid u_{2}(z)>M_{2}\right\}$. Hence the function

$$
\begin{array}{ll}
u_{3}(z)=u_{2}(z)-M_{2}, & z \in D_{4}, \\
u_{3}(z)=0, & z \notin D_{4},
\end{array}
$$

is non-negative, non-constant and subharmonic in the plane. In $D_{4}, u_{3}$ is positive and harmonic, and possesses there a local conjugate $v$ such that with $\alpha=\beta-\varepsilon$, the function

$$
F(z)=z^{1-\alpha} \exp \left(u_{3}+i v\right)
$$

is single-valued in $D_{4}$. Hence we can apply Lemma 1 to $u_{3}$ to deduce that $D_{4}$ contains 
a neighbourhood of infinity. But since $D_{4} \subset G_{1}$, it follows that $G$ contains a punctured neighbourhood of $z_{1}$, so that $z_{1}$ is a regular point of $f$ and so there exists a positive integer $k$ and $a_{k}$ unequal to zero such that

$$
\begin{aligned}
f(\zeta)-f\left(z_{1}\right) \sim a_{k}\left(\zeta-z_{1}\right)^{k} & \text { as } \quad \zeta \rightarrow z_{1}, \\
f\left(z_{1}+1 / z\right)-f\left(z_{1}\right) \sim a_{k} z^{-k} & \text { as } \quad z \rightarrow \infty .
\end{aligned}
$$

This together with (1.2) and (3.2) implies that

$$
u(z)=-k \log |z|-\log \mu(1 /|z|)+O(1) \leqq o(\log |z|)
$$

as $z \rightarrow \infty$, which contradicts the result $\beta>0$. So in any case $u_{1}$ is bounded above in a neighbourhood of $z_{1}$ in $\bar{G}$.

We want to show that $u_{1}(z) \leqq 0$ in $G$, since in view of Lemma 2 this clearly completes the proof of Theorem 1. If $u_{1}$ is bounded above in $G$, this follows from the maximum principle. Suppose then that $u_{1}$ is not bounded above in $G$, and let $D$ be a component of $\left\{z \mid u_{1}(z)>0\right\}$. Since $u_{1}$ is bounded above in any compact subset of $\bar{G}$, as we proved above, the set $D$ must be unbounded.

We set $u(z)=u_{1}(z)$ in $D, u(z)=0$ outside $D$. To make $u$ subharmonic, we may have to redefine $u$ at $z_{1}$, cf. [1, p. 248]. Then we apply Lemma 1 to $u$. We assume that (1.4) or (1.5) holds. Without loss of generality we may assume that $z_{1}=0$. Thus we obtain

$$
\sup _{|z|=r, z \in D} \log |f(z)-f(0)| \sim(\beta+A) \log r
$$

as $r \rightarrow \infty$. Suppose now that $A<1$. We choose $\varepsilon$ such that $0<\varepsilon<\beta$ and $A+\varepsilon<1$, and $R$ such that

and set

$$
\log \mu(|z|)<(A+\varepsilon) \log |z|,|z| \geqq R,
$$

$$
u_{2}(z)=\log |f(z)-f(0)-(A+\varepsilon) \log | z \mid-M_{3}, \quad z \in D,
$$

where $M_{3} \geqq 0$ and $M_{3}$ is so large that $u_{2}(z) \leqq 0$ if $z \in D$ and $|z|=R$. Hence $u_{2}$ has negative boundary values in $D \cap\{|z|>R\}$, but $u_{2}$ is unbounded above in $D \cap\{|z|>R\}$. Therefore we can find an unbounded component $D_{1}$ of the set $\left\{z \in D|z|>R, u_{2}(z)>0\right\}$, and we define $u_{3}(z)=u_{2}(z)$ in $D_{1}, u_{3}(z)=0$ outside $D_{1}$.

The function $u_{3}$ is subharmonic in the plane, harmonic in $D_{1}$, and possesses there a local conjugate. We may apply Lemma 1 to $u_{3}$ with $\alpha=1-A-\varepsilon$ to deduce that $D_{1}$ and so $G$ contains a neighbourhood of $\infty$. Hence by (1.4), $f$ remains bounded at $\infty$, so that $u_{1}$ is bounded above, which is contrary to our assumption. This proves Theorem 1 if $A<1$.

Suppose that $A=1$. We shall soon show that then $\mu(t)=M t, M>0$. Since (1.5) holds, Theorem 1 follows now from [1, Theorem 1].

If $A=1$, then $B=1$. Since

$$
\eta(t)=\log \mu\left(e^{t}\right)-\log \mu(1)
$$


is concave with $\eta(0)=0$, we have

$$
\begin{gathered}
0=2 \eta(0) \geqq \eta(t)+\eta(-t), \quad t>0, \\
\eta(t) / t \leqq \eta(-t) /(-t), \quad t>0 .
\end{gathered}
$$

Further $\eta(t) / t$ decreases to 1 and $\eta(-t) /(-t)$ increases to 1 as $t$ increases from 0 to $\infty$. Hence $\eta(t) \equiv t, \mu(t) \equiv t \mu(1)$. This proves Theorem 1 if $A=1$.

Suppose finally that (1.6) holds for some $q>0$ but that the conclusion of Theorem 1 fails. Suppose that (1.1) fails for some $z_{1} \in \partial G, z_{2} \in G$. We can still deduce that $u_{1}$, given by (3.1), is bounded above in a neighbourhood of $z_{1}$ in $\bar{G}$ and that (2.1) is satisfied with $u=u_{1}$. Since $u_{1}$ must be unbounded above in $G$, we obtain as before from Lemma 1 that $G$ contains a neighbourhood of infinity. Now (1.6) implies that $f$ has a pole at infinity.

If (1.1) fails for some $z_{1}, z_{2} \in G$ but not for any $z_{1} \in \partial G, z_{2} \in \bar{G}$, then we take these $z_{1}, z_{2} \in G$, set $h=z_{2}-z_{1}$ and $g(z)=f(z)-f(z+h)$. If $R$ is defined as in the proof of Lemma 2 for $t=|h|$, then the function $u$ given by (2.6) must be unbounded in $G_{1}$, given by (2.5). Since $u$ satisfies (2.1), we deduce from Lemma 1 that $G$ contains a neighbourhood of infinity. Now (1.6) implies that $f$ has a pole at infinity. Theorem 1 is proved.

Remark. After this paper had been written, I was informed that there is a recent preprint of Tamrazov containing results similar to those in this paper.

\section{References}

[1] Gehring, F. W., W. K. Hayman, and A. Hinkkanen: Analytic functions satisfying Hölder conditions on the boundary. - J. Approx. Theory 35, 1982, 243-249.

[2] Tamrazov, P. M.: Contour and solid structure properties of holomorphic functions of a complex variable. - Russian Math. Surveys 28:1, 1973, 141-173.

Imperial College

Department of Mathematics

London SW7 2BZ

England

Received 13 February 1984
Current address:

University of Michigan

Department of Mathematics

Ann Arbor, Michigan 48109

USA 